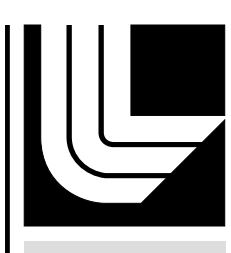

LA W REN CE LIVERM ORE N A TIO NAL LABORATORY

Secondary shock formation in xenon-nitrogen mixtures

J.F. Hansen, M.J. Edwards, D.H. Froula, A. D. Edens, G. Gregori, T. Ditmire

October 17, 2006

Physics of Plasmas 
This document was prepared as an account of work sponsored by an agency of the United States Government. Neither the United States Government nor the University of California nor any of their employees, makes any warranty, express or implied, or assumes any legal liability or responsibility for the accuracy, completeness, or usefulness of any information, apparatus, product, or process disclosed, or represents that its use would not infringe privately owned rights. Reference herein to any specific commercial product, process, or service by trade name, trademark, manufacturer, or otherwise, does not necessarily constitute or imply its endorsement, recommendation, or favoring by the United States Government or the University of California. The views and opinions of authors expressed herein do not necessarily state or reflect those of the United States Government or the University of California, and shall not be used for advertising or product endorsement purposes. 


\title{
Secondary shock formation in xenon-nitrogen mixtures
}

\author{
J.F. Hansen, M.J. Edwards, and D.H. Froula \\ Lawrence Livermore National Laboratory, Livermore, CA 94550, USA
}

A. D. Edens

Sandia National Labs, Albuquerque, NM 87185, USA

G. Gregori

LRC - Rutherford Appleton Laboratory, Chilton, Didcot OX11 OQX, UK

T. Ditmire

University of Texas at Austin, Austin, TX 78712, USA

(Date textdate; Received textdate; Revised textdate; Accepted textdate; Published textdate)

\begin{abstract}
The expansion of shock waves have been studied in mediums with different opacities and heat capacities, varied in systematic ways by mixing xenon with nitrogen keeping the mass density constant. An initial shock is generated through the brief ( $5 \mathrm{~ns})$ deposition of laser energy $(5 \mathrm{~J})$ on the tip of a pin surrounded by the xenon-nitrogen mixture. The initial shock is spherical, radiative, with a high Mach number, and it sends a supersonic radiatively driven heat wave far ahead of itself. The heat wave rapidly slows to a transonic regime and when its Mach number drops to $\sim 2$ with respect to the downstream plasma, the heat wave becomes of the ablative type, driving a second shock ahead of itself to satisfy mass and momentum conservation in the heat wave reference frame. The details of this sequence of events depend, among other things, on the opacity and heat capacity of the surrounding medium. Second shock formation is observed over the entire range from $100 \%$ Xe mass fraction to $100 \% \mathrm{~N}_{2}$. The formation radius of the second shock as a function of Xe mass fraction is consistent with an analytical estimate.
\end{abstract}




\section{INTRODUCTION}

We previously reported on the experimental discovery of secondary shock formation ahead of strongly radiative blast waves in Xe $[1,2]$. The process can be summarized as follows (c.f. Fig. 1): an expanding spherical shock (which we will refer to as s1) is initially fast enough to radiate very strongly, with an inverse Boltzmann number $B o^{-1}=\sigma T^{4} / v_{s} \rho_{0} c_{v} T \sim 50$ (where $T$ is the shock temperature, $v_{s}$ is the shock speed, and $\rho_{0}$ is the density of the ambient gas). The radiation mean free path in the cold ambient gas ahead of s1 is relatively short, resulting over time in the formation of a supersonic, radiative heat wave (RHW), which propagates in advance of s1. As s1 continually slows down, it radiates less and less, and the radiated power soon drops below the rate at which s1 sweeps up energy from gas heated by RHw, i.e., the energy loss rate becomes negative. At this time, most of the energy that was originally in $\mathrm{S} 1$ has been radiated to RHW, and RWH is far ahead of S1. However, the velocity of RHW has also been diminishing rapidly because of expansion and a rapidly weakening driving source. Eventually RHW becomes transonic and gives birth to a second shock wave (s2). RHW then falls behind $\mathrm{s} 2$, which itself is too slow to be radiative. $\mathrm{s} 1$ continues to weaken as it propagates in the downstream material of $\mathrm{s} 2$ and soon dissipates. After $\mathrm{s} 2$ has roughly doubled its radius, it is no longer influenced by the details of how it was formed, and the shock trajectory closely assumes that of a self-similar Sedov-Taylor blast wave [4-7].

The motivation behind our experiments (and behind many other experiments in laboratories around the world [8-15]) is an interest in astrophysical shocks which have high Mach numbers and which may be radiative [16], e.g., shocks originating in supernova (SN) explosions $[9,17-20]$. The nature of these shocks is important to understand as the shocks mix up interstellar matter and thus affect mass-loading, stellar formation [21-23], and the history of the Milky Way and other galaxies. Although the motivation for our experiments was an interest in astrophysical shocks, we should point out that the character of these laboratory shocks may be different from any of astrophysical importance, and that secondary shock formation has not been observed or postulated in astrophysical shocks to date. 


\section{BACKGROUND}

The radiative nature of a shock, coupled with the optical opacity and heat capacity of its surroundings, largely determines the evolution of the shock and its rate of expansion. The energy loss rate can be quantified (in units of how much energy the expanding shock sweeps up) by the dimensionless number $[3,7] \varepsilon=-(\mathrm{d} E / \mathrm{d} t)\left(2 \pi \rho_{0}\right)^{-1} r_{s}^{-2}\left(\mathrm{~d} r_{s} / \mathrm{d} t\right)^{-3}$, where $E$ is the total energy content and $r_{s}$ the shock radius. In a strongly radiative case, in which radiation escapes to infinity, the incoming kinetic energy swept up by a shock is entirely radiated away $(\varepsilon \rightarrow 1)$ and the shocked material collapses to a thin shell directly behind the shock. For an adiabatic case $(\varepsilon=0)$ once the shock has swept up more mass than what was initially present, the shock could be regarded as without characteristic length or time scales, and one would expect the well-known self-similar motion of a Sedov-Taylor blast wave [4-7], $r_{s} \propto t^{\alpha}$, where the exponent $\alpha=2 / 5$. In a case where radiation removes energy from the shock in an optically thin environment, analytical and numerical studies predict a slower shock expansion, such as $\alpha=2 / 7$ (the "pressure-driven snowplow"), $\alpha=1 / 4$ (the "momentum-driven snowplow"; the shock is simply coasting) [16, 24], and $2 / 7<\alpha<2 / 5$ (the thermal energy of the shocked gas is not completely radiated away) $[3,7]$.

In a case where the environment is not optically thin, radiation is reabsorbed in the upstream material and if the shock is travelling fast enough a supersonic, radiative heat wave (RHW) breaks away from the shock in a situation analogous to a supercritical shock wave [6]. This case was studied by Reinicke and Meyer-ter-Vehn [25], who showed that the shock and RHW will coexist and eventually propagate as $r \propto t^{\alpha}$ where $\alpha$ is larger for the shock. This means that the shock would eventually catch RHW, after which a second state is obtained in which RHW is of the ablative type and the shock moves in a classical Sedov-Taylor trajectory with $\alpha=2 / 5$.

In our earlier experiments [2] we came across an additional possibility for optically thin environments, namely that prior to the shock catching RHW, the latter enters a transonic regime, stalls, and generates a second shock (s2). We showed that an analytical estimate for the formation radius of $\mathrm{s} 2$ can be obtained from a standard result of heat front physics $[6,26,27]$, using the steady, $1 \mathrm{D}$ fluid equations for conservation of mass $\rho_{1} u_{1}=\rho_{2} u_{2}$ and momentum $p_{1}+\rho_{1} u_{1}^{2}=p_{2}+\rho_{2} u_{2}^{2}$ in the frame of RHW, where subscript 1 denotes the region ahead of RHW, and subscript 2 denotes the region behind RHW. We have dropped 
the time-dependent terms in the conservation equations as these are neglible compared to the spatial gradient terms; the front thickness of RHW is very small in comparison to the RHW radius, so even though RHW moves with variable (slowing) velocity, physical quantities remain practically unchanged during the small time required to traverse the front width $[6,25]$. This is particularly true as RHW sharpens up just prior to generating s2. Assuming an ideal gas (so that $p=\rho c^{2}$ where $c$ is the speed of sound) we combine the conservation equations to obtain

$$
\frac{\rho_{2}}{\rho_{1}}=\frac{c_{1}^{2}+u_{1}^{2} \pm \sqrt{\left(c_{1}^{2}+u_{1}^{2}\right)^{2}-4 c_{2}^{2} u_{1}^{2}}}{2 c_{2}^{2}} .
$$

A supersonic $\left(u_{1}>c_{1}\right)$ RHW and a real compression $\eta \equiv \rho_{2} / \rho_{1}$ requires

$$
u_{1} \geq c_{2}+\sqrt{c_{2}^{2}-c_{1}^{2}} \approx 2 c_{2}
$$

(where the approximation is valid because the temperature behind RHW is much higher than the temperature before it), i.e., requires the mixed Mach number

$$
M \equiv \frac{u_{1}}{c_{2}} \geq 2
$$

Once the Mach number drops to 2, RHW can no longer fulfill Eq. 1, and s2 forms at RHW. $\mathrm{s} 2$ immediately moves ahead of RHW and acts to slow down $u_{1}$ so that RHW is now subsonic, satisfying

$$
u_{1} \leq c_{2}-\sqrt{c_{2}^{2}-c_{1}^{2}} \approx \frac{c_{1}^{2}}{2 c_{2}} .
$$

To estimate the radius $r_{h}$ of RHW at the moment when its Mach number is 2 , and thus the formation radius of $\mathrm{s} 2$, we can assume a radiative conductivity (with units $\mathrm{J} \mathrm{m}^{-1} \mathrm{~K}^{-1} \mathrm{~s}^{-1}$ ) of the ambient gas of the form

$$
\chi=\chi_{0} \rho^{a} T^{b}
$$

and use Barenblatt's solution for an instantaneous point release of energy [25, 28]:

$$
r_{h}(t)=r_{0} t^{\delta}
$$


where

$$
\begin{aligned}
r_{0} & =\left(K_{1}^{b} K_{2}\right)^{\delta} \\
\delta & =\frac{1}{3 b+2} \\
K_{1} & =\frac{\gamma-1}{2 \pi B\left(\frac{3}{2}, 1+\frac{1}{b}\right)} \frac{E}{\rho_{0}} \\
K_{2} & =\frac{2 \chi_{0}(\gamma-1)}{\Gamma^{b+1} \rho_{0}^{1-a}} \frac{1}{b \delta},
\end{aligned}
$$

where $\gamma$ is the adiabatic index, $B(x, y)$ is the beta function, and $\Gamma$ is the gas constant from the ideal gas equation of state

$$
(\gamma-1) e=\Gamma T
$$

where $e$ is the specific internal energy [with units $\mathrm{J} / \mathrm{kg}$; the heat capacity $c_{v}=\Gamma /(\gamma-1)$. The RHW Mach number is obtained from

$$
u_{1}=\frac{\mathrm{d} r_{h}}{\mathrm{~d} t}
$$

and

$$
c_{2}(r=0)=\left(K_{1}^{2} K_{2}^{-3} t^{-3}\right)^{\delta / 2}=K_{1}^{\delta} K_{2}^{-3 \delta / 2}\left(r_{h} / r_{0}\right)^{-3 / 2} .
$$

Using the sound speed at $r=0$ is a reasonable approximation as the temperature profile inside RHW is quite flat $[2,25,28]$. We should also point out that using Barenblatt's solution to estimate the RHW radius assumes that radiation can be treated in the diffusion approximation. In the earliest expansion phase this is not the case, but Barenblatt's solution turns out to be a reasonable approximation when the wave has cooled somewhat and has large enough optical depth. For pure Xe this would be at $t \approx 20 \mathrm{~ns}$; at this time the mixed Mach number $M \approx 9$, the RHW radius $r_{h} \approx 4 \mathrm{~mm}$, compared to $r_{s} \approx 1.4 \mathrm{~mm}$, and only about one eighth the total energy still resides in the initial shock (the rest is in the RHW). For pure Xe with $\rho_{0} \sim 10^{-5} \mathrm{~g} / \mathrm{cm}^{3}, \chi_{0}=1 \times 10^{-44} \mathrm{~kg}^{-1.2} \mathrm{~K}^{-11} \mathrm{~m}^{-5.6} \mathrm{~s}^{-3}, a=-2.2, b=10$, and $E=5 \mathrm{~J}$, this analytical estimate says that $K_{1}=1.79, K_{2}=5.0 \times 10^{-61}$, and that the RHW Mach number drops to Mach 2 when $r_{h} \approx 10 \mathrm{~mm}$ (and the speed of sound behind RWH is $c_{2} \approx 1.4 \mathrm{~km} / \mathrm{s}$ ), in reasonable agreement with the experiment where we first observed $\mathrm{s} 2$ with $r \approx 12 \mathrm{~mm}[2]$.

We report here on a new series of experiments using mixtures of $\mathrm{Xe}$ and $\mathrm{N}_{2}$. The goal of these experiments was to further study the previously unreported second shock, to see what 
the effect is of changing the radiative conductivity (or equivalently the opacity) and the heat capacity, and to see if the above analytical estimate holds under a range of conditions.

\section{EXPERIMENT SET-UP AND DIAGNOSTICS}

We create spherically expanding blast waves in the following fashion: a high-energy infrared pulsed laser (1064 nm wavelength) is focused onto the tip of a solid (stainless steel) pin surrounded by a Xe/ $\mathrm{N}_{2}$ mixture with a density of $\rho_{0}=3.6 \times 10^{-5} \mathrm{~g} / \mathrm{cm}^{3}$. The laser pulse is $5 \mathrm{~ns}$ in duration with energy $E_{l} \approx 5 \mathrm{~J}$ (the exact energy fluctuates slightly from shot to shot but is measured and recorded). Most of this energy is deposited in pin material which then becomes very hot and expands rapidly, pushing at the surrounding gas, setting up a strong, radiative initial shock (s1), see Fig. 2. We estimate that most ( $\sim 98 \%)$ of our relatively modest $E_{l} \approx 5 \mathrm{~J}$ contribues to plasma expansion and is not reflected, based on scaling from earlier experiments [2] with a much higher $E_{l} \approx 100 \mathrm{~J}$ where only $\sim 25 \%$ of the energy was deposited and using the formula [29] $R(1-R)=\varepsilon \exp (G(1-R))$ where $R$ is the reflectivity, $\varepsilon \approx 10^{-9}$ and assuming $G \propto \sqrt{E_{l}}$. At the end of the laser pulse ( $t=5 \mathrm{~ns}$ ), s1 is traveling in excess of $60 \mathrm{~km} / \mathrm{s}$ and is (at least for mixtures with a significant fraction of Xe) strongly radiative. When the radius $r_{s} \sim 0.4 \mathrm{~mm}, \mathrm{~s} 1$ has swept up enough material that the details of its initial conditions are unimportant. Radiation from $\mathrm{s} 1$ heats the surrounding gas. The (inverse) Boltzmann number $B o^{-1} \gtrsim 50$ initially, i.e., s1 radiatively drives a supersonic heat wave that travels rapidly outward, leaving a large separation between s1 and the radiatively driven heat wave. With time, $\mathrm{s} 1$ slows and its ability to radiate efficiently quickly decreases, while the heat wave becomes a radiative heat wave (RHW) of the diffusive type. Also, $\mathrm{s} 1$ is traveling into the counter pressure of hot RHW plasma, which is becoming comparable to the ram pressure; the Mach number of s1 drops rapidly, and the post-shock compression reduces correspondingly. The Mach number for RHW is also decreasing, and when it reaches $\sim 2$, RHW stalls and creates $\mathrm{S} 2$, with a radius measured in earlier experiments in pure Xe of $\sim 12 \mathrm{~mm}$. After this time, s1 continues to weaken until it dissipates, while s2 is essentially non-radiative and once it has swept up enough mass (doubled its initial radius), it propagates like $r_{s} \propto t^{2 / 5}$.

To image s1 and s2 on spatial scales up to $\sim 5 \mathrm{~cm}$, we used two lenses in a telescope configuration and a gated, single-frame, high-speed CCD camera (2 ns gate), along with 
a low energy, green laser pulse ( $\lambda=532 \mathrm{~nm}$ wavelength, $15 \mathrm{~ns}$ duration) as a backlighter. We employed a schlieren technique with a vertical knife edge at the telescope focal point to remove light which had not been deflected by the plasma. With this method, image brightness corresponds to the spatial derivative of plasma electron density in the horizontal direction, so that vertical structures in the plasma are readily seen. A monochromatic filter was placed in front of the camera to prevent damage to the CCD (damage occured in our previous experiments), with the disadvantage that glow from the heated plasma could not be seen in this experiment (c.f. Fig. 1).

A spectrometer was used to obtain spectral line intensity as a function of position (ahead of and behind the blast wave), which was then Abel inverted to get spectral line intensity as a function of radius. An estimate of temperature as a function of radius was then calculated using the Saha equation from pair-wise line ratios. This was done in pure $\mathrm{N}_{2}$ using two NII lines (399.5 nm and $444.70 \mathrm{~nm})$ and two NIII lines (451.485 nm and $463.413 \mathrm{~nm})$ and in pure Xe using three XeII lines, $441.48 \mathrm{~nm}, 446.22 \mathrm{~nm}$, and $460.3 \mathrm{~nm}$. These measurements were done at relatively late experimental times (e.g., around the formation time of s2), when the electron-ion collisional time scale is about one order of magnitude shorter than the characteristic time scale for RHW, justifying using the Saha equation to obtain reasonable temperature estimates.

\section{RESULTS}

Keeping the density constant (by keeping the partial pressures $p_{X e} m_{X e}+p_{N_{2}} m_{N_{2}}=C$ where $C$ is a constant), we varied the composition of the ambient gas from $100 \%$ Xe (by mass) to $100 \% \mathrm{~N}_{2}$ and tracked the formation of the second shock. We found that the higher the fraction of $\mathrm{N}_{2}$, the smaller the formation radius became (and the sooner the second shock forms). This trend is shown in Fig. 3, where each column represent a certain mixture of Xe and $\mathrm{N}_{2}$, and time runs toward the bottom of each column. In the top row of images, we only see the initial shock. As we follow each column down, the second shock forms, and the initial shock dissipates; this should be particularly obvious around the middle of each column. The bottom row shows images where only the second shock can be seen.

It is worthwhile noting the experimental difficulty in observing the second shock. Previous experiments with similar laser energies and gas densities have not observed secondary 
shock formation, and this may be because of any of the following: (a) the experiment was studying radiative shocks, so images were obtained only relatively early in time, while s1 is

still radiative, (b) the experiment was studying blast waves, so images were only obtained relatively late in time, to ensure that a stable Sedov-Taylor blast-wave had formed, (c) images were too sparse in time, i.e., the sequence of images shows s1 in the first few images, then switches to $\mathrm{S} 2$ without capturing the moments when both exist simultaneously, and the experimenter believed - quite naturally - that the same shock was observed in all the images, (d) the schlieren technique was not sensitive enough. The latter condition is one that we struggled with. When the knife-edge position was not carefully calibrated, we obtained images for the intermediate times (when both shocks exist) that simply show no shock at all (both shocks are too weak to perturb the plasma enough to overcome the crudely positioned knife-edge).

Figure 3 can be represented in a bar plot, where each bar represents an uncertainty in the formation radius of the second shock; the lower end of each bar is the radius of s1 in an image where the second shock cannot yet be seen, while the upper end is the radius of s2 in the earliest image for each mixture in which we can see both s1 and s2. We have measured all shock radii and are showing this data in Fig. 4. Also shown in Fig. 4 are second shock formation radii from our previous work in pure Xe [2].

\section{DISCUSSION}

Barenblatt's solution assumes a radiative heat conductivity which is a function of temperature (and density), but the heat capacity is implicitly assumed to be constant. This means that, at best, Barenblatt's solution will only be an approximation to real gases with non-constant heat capacities. To use Barenblatt's solution, a constant heat capacity was based, for each mixture, on a temperature estimated from the emission spectroscopy data; $T \sim 3.2 \mathrm{eV}$ for pure $\mathrm{Xe}, \mathrm{T} \sim 5.5 \mathrm{eV}$ for pure $\mathrm{N}_{2}$, and temperatures interpolated between these two values for the mixtures. The following steps illustrate our method in estimating when the RHW becomes transonic and s2 forms:

1. The adiabatic index $\gamma$ for each gas mixture is assumed unknown and is estimated from 
Sedov-Taylor's formula for a self-similar blast wave:

$$
r_{s}=\left(\frac{75}{16 \pi} \frac{(\gamma-1)(\gamma+1)^{2}}{3 \gamma-1}\right)^{\frac{1}{5}}\left(\frac{E}{\rho_{0}}\right)^{\frac{1}{5}} t^{\frac{2}{5}}
$$

where we use the measured blast-wave radius $r_{s}$ from an image obtained at a very late time $t$ so that the second shock is well-developed past the point where its initial conditions matter, in addition to the recorded laser energy $E$ and measured density $\rho_{0}$. We typically find that $\gamma \approx 1.05$, consistent with previously published values by Grun et al.

2. The gas constant $\Gamma$ is calculated from Eq. 11 using the adiabatic index $\gamma$ from step 1, an initial estimate of a representative temperature, and a specific internal energy

$$
e=e_{0} \rho^{f} T^{g}
$$

with parameters $e_{0}, f$, and $g$ calculated using the LASNEX code [31] and listed in Table I.

3. The various parameters appearing in Barenblatt's solution (in Eqs. 7-10) are calculated using the adiabatic index $\gamma$ from step 1, the gas constant $\Gamma$ from step 2, and a radiative conductivity from Eq. 5 using the parameters $\chi_{0}, a$, and $b$, obtained for pure nitrogen from the OPAL tables [32] and for the mixtures calculated according to the methods in the STA code [33] using the Lawrence Livermore VISTA code, and listed in Table I.

4. The RHW radius $r_{h}$ can be solved for analytically using Eqs. 12-13 above (but the expression is too complicated to cast any light on the physics and is not included here). We then set the mixed mach number $M=u_{1} / c_{2}=2$ to obtain the radius $r_{2}=\left.r_{h}\right|_{M=2}$ when the second shock forms. [Note that if the parameter $b \gg 1$ (which is the case here) the simple estimate

$$
r_{2} \approx\left(\frac{K_{1}^{b} K_{2}}{6 b}\right)^{1 / 3 b}
$$

can be used to $15 \%$ or better accuracy.]

As a final check one could calculate the temperature

$$
T_{\text {Barenblatt }}=\frac{c_{2}^{2}}{\Gamma} .
$$

inside RHW and compare to the initial estimate of $T$ obtained from our spectrometer data. We find that a temperature calculated from Eq. 17 agrees quite well with the initial estimate and the discrepancy is less than $0.6 \mathrm{eV}$ in all cases. If we go back and use $T_{\text {Barenblatt }}$ in step 2 we get less than a $4 \%$ difference in our final answer for $r_{2}$. 
The calculated values for $r_{2}$ in seven different mixes of Xe and $\mathrm{N}_{2}$ are shown alongside the experimental data in Fig. 4. Higher fractions of $\mathrm{N}_{2}$ result in smaller $\mathrm{s} 2$ formation radii (primarily due to the higher heat capacity), and generally the agreement between analytical estimate and the experimental data is quite good.

In the astrophysical case, second shocks are unlikely to form around the most energetic events, such as a supernovae (with say $E=10^{46} \mathrm{~J}$ or $10^{53} \mathrm{erg}$ ). Either one chooses bremstrahlung $\left(\chi_{0}=8.3 \times 10^{-28} \mathrm{~kg}^{3} \mathrm{~K}^{-15 / 2} \mathrm{~m}^{-5} \mathrm{~s}^{-3} ; a=-2 ; b=6.5\right)$ or compton scattering $\left(\chi_{0}=7.6 \times 10^{-6} \mathrm{~kg}^{2} \mathrm{~K}^{-4} \mathrm{~m}^{-2} \mathrm{~s}^{-3} ; a=-1 ; b=3\right)$ or some other physical process to limit the radiative conductivity, one finds that in interstellar hydrogen $\left(\rho=1 \mathrm{atom} / \mathrm{cm}^{3}\right)$, a second shock would not form in a time shorter than the lifetime of the universe. If this energy was released in a denser environment, say $\rho=10^{5}$ atoms $/ \mathrm{cm}^{3}$ (equivalent to the higher density regions in the horsehead nebula), RHW would slow to Mach 2 in a shorter time, but would still have a transition radius of at least 500 lightyears, larger than any astrophysical object with this density (the horsehead nebula is about 25 lightyears across). In other words, if one wishes to observe second shock formation in interstellar space, less energetic events are better candidates. An energy release of $E=10^{35} \mathrm{~J}$ in a density of $\rho=10^{5}$ atoms $/ \mathrm{cm}^{3}$ would produce a second shock with a formation radius of about one lightyear.

\section{SUMMARY}

We have varied in a systematic way the opacity and heat capacity of the gas into which a spherical shock wave expands (by mixing Xe with $\mathrm{N}_{2}$ keeping the mass density constant) and measured when a second shock forms ahead of the initial shock. The formation radius of the second shock as a function of Xe mass fraction is consistent with an analytical estimate where the expansion of a radiatively driven heat wave is estimated using Barenblatt's solution and the heat wave then stalls as its Mach number drops to $\sim 2$.

\section{ACKNOWLEDGEMENTS}

We thank Dwight Price and the staff at the Janus facility (where the experiments were conducted) for their valuable assistance.

This work was performed under the auspices of the U. S. Department of Energy by 
the University of California, Lawrence Livermore National Laboratory under Contract No. W-7405-Eng-48.

[1] J. F. Hansen, M. J. Edwards, D. Froula, G. Gregori, A. Edens, and T. Ditmire, Astrophysics and Space Science, 298, 61 (2005).

[2] J. F. Hansen, M. J. Edwards, D. H. Froula, G. Gregori, A. D. Edens, and T. Ditmire, Phys. Plasmas, 13, 1 (2006).

[3] E. Cohen, T. Piran, and R. Sari, Astrophys. J., 509, 717 (1998).

[4] G. I. Taylor, Proc. R. Soc. London A, 201, 159 (1950).

[5] L. I. Sedov, Similarity and Dimensional Methods in Mechanics (Academic, New York, 1959).

[6] Y. B. Zeldovich and Y. P. Raizer, Physics of Shock Waves and High-Temperature Hydrodynamic Phenomena (Academic, New York, 1966).

[7] Edison Liang and Katherine Keilty, 2000, Astrophys. J., 533, 890 (2000).

[8] J. C. Bozier, G. Thiell, J. P. LeBreton, S. Azra, M. Decroisette, and D. Schirmann, Phys. Rev. Lett. 57, 1304 (1986).

[9] B. A. Remington, D. Arnett, R. P. Drake, and H. Takabe, Science, 284, 1488 (1999).

[10] D. Ryutov, R. P. Drake, J. Kane, E. Liang, B. A. Remington, and W. M. Wood-Vasey, Astrophys. J., 518, 821 (1999).

[11] K. Shigemori, T. Ditmire, B. A. Remington, V. Yanovksy, D. Ryutov, K. G. Estabrook, M. J. Edwards, A. J. MacKinnon, A. M. Rubenchik, K. A. Keilty, and E. Liang, Astrophys. J., 533, 159 (2000).

[12] H. F. Robey, J. O. Kane, B. A. Remington, R. P. Drake, O. A. Hurricane, H. Louis, R. J. Wallace, J. Knauer, P. Keiter, D. Arnett, and D. D. Ryutov, Phys. of Plasmas, 8, 2446 (2001).

[13] P. A. Keiter, R. P. Drake, T. S. Perry, H. F. Robey, B. A. Remington, C. A. Iglesias, R. J. Wallace, and J. Knauer, Phys. Rev. Lett., 89, 165003-1 (2002).

[14] X. Fleury, S. Bouquet, C. Stehle, M. Koenig, D. Batani, A. Benuzzi-Mounaix, J.-P. Chieze, N. Grandjouan, J. Grenier, T. Hall, E. Henry, J.-P. Lafon, S. Leygnac, V. Malka, B. Marchet, H. Merdji, C. Michaut, and F. Thais, Laser and Particle Beams, 20, 263 (2002).

[15] S. Bouquet, C. Stehle, M. Koenig, J.-P. Chieze, A. Benuzzi-Mounaix, D. Batani, S. Leygnac, X. Fleury, H. Merdji, C. Michaut, F. Thais, N. Grandjouan, T. Hall, E. Henry, V. Malka, and 
J.-P.J. Lafon, Phys. Rev. Lett., 92, 225001-1 (2004).

[16] J. M. Blondin, E. B. Wright, K. J. Borkowski, and S. P. Reynolds, Astrophys. J., 500, 342 (1998).

[17] E. Müller, B. Fryxell, and D. Arnett, Astron. Astrophys., 251, 505 (1991).

[18] J. I. Reed, J. J. Hester, A. C. Fabian, and P. F. Winkler, Astrophys. J., 440, 706 1995).

[19] G. Sonneborn, C. S. J. Pun, R. A. Kimble, T. R. Gull, P. Lundqvist, R. McCray, P. Plait, A. Boggess, C. W. Bowers, A. C. Danks, J. Grady, S. R. Heap, S. Kraemer, D. Lindler, J. Loiacono, S. P. Maran, H. W. Moos, and B. E. Woodgate, Astrophys. J. Lett., 492, L139 (1998).

[20] N. Bartel, M. F. Bietenholz, M. P. Rupen, A. J. Beasley, D. A. Graham, V. I. Altunin, T. Venturi, G. Umana, W. H. Cannon, and J. E. Conway, Science, 287, 112 (2000).

[21] C. F. McKee and B. T. Draine, Science, 252, 397 (1991).

[22] D. A. Allen and M. G. Burton, Nature, 363, 54 (1993).

[23] R. I. Klein and D. T. Woods, Astrophys. J., 497, 777 (1998).

[24] C. F. McKee and J. P. Ostriker, Astrophys. J., 218, 148 (1977).

[25] P. Reinicke and J. Meyer-ter-Vehn, Phys. Fluids A, 3, 1807 (1991).

[26] D. Mihalas and B. Weibel Mihalas, Foundations of Radiation Hydrodynamics (Oxford University Press, Oxford, 1984).

[27] S. P. Hatchett, UCRL-JC-108348 Ablation Gas Dynamics of Low-Z Materials Illuminated by Soft X-Rays (Lawrence Livermore National Laboratory, CA, 1991). Copies may be obtained from the National Technical Information Service, Springfield, VA 22161.

[28] G. I. Barenblatt, Similarity, Self-Similarity and Intermediate Asymptotics (Consultants Bureau, New York, 1979).

[29] C. L. Tang, J. Appl. Phys., 37, 2945 (1966).

[30] J. Grun, J. Stamper, C. Manka, J. Resnick, R. Burris, J. Crawford, and B. H. Ripin, Phys. Rev. Lett., 66, 2738 (1991).

[31] G. B. Zimmerman and W. L. Kruer, Comments Plasma Phys. Controlled Fusion, 2, 51 (1975).

[32] C. A. Iglesias and F. J. Rogers, Astrophys. J., 464, 943 (1996).

[33] A. Bar-Shalom, J. Oreg, W. H. Goldstein, D. Shvarts, A. Zigler, Phys. Rev. A, 40, 3183 (1989). 


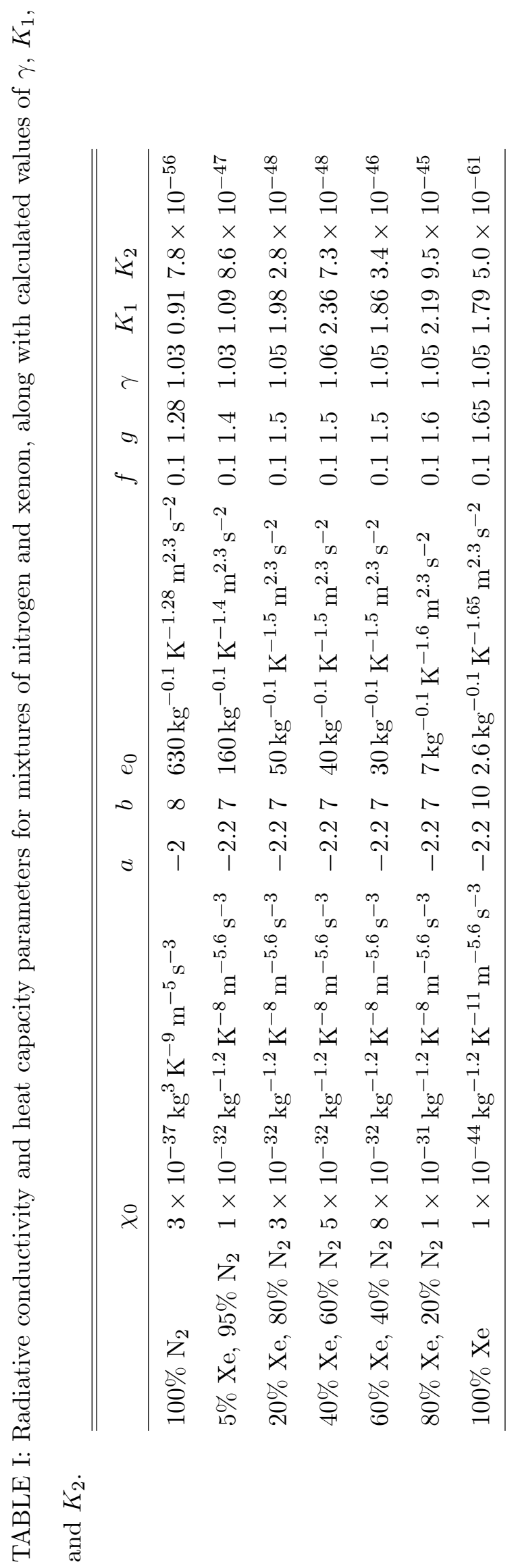




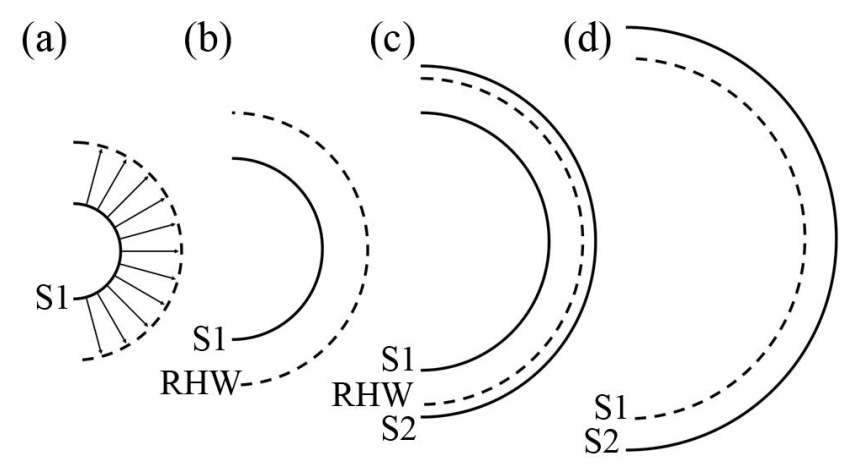

FIG. 1: Sequence of events (time increasing from left to right): (a) A spherical shock, s1, radiatively drives a heat wave far ahead of itself. (b) As s1 expands it slows down and it radiates less and less. The radiative heat wave, $\mathrm{RHW}$, also slows down and its expansion rate can be estimated using Barenblatt's solution. (c) S1 gains on RHW, but before it catches up, RHW becomes transonic and gives birth to a second shock wave, $\mathrm{s} 2$. RHW immediately falls behind $\mathrm{s} 2$, which itself is too slow to be radiative. (d) S1 continues to weaken as it propagates in the downstream material of s2 and soon dissipates. In the experiment, shocks that are drawn here with solid curves are visible in the schlieren images, features drawn with dashed curves are not.

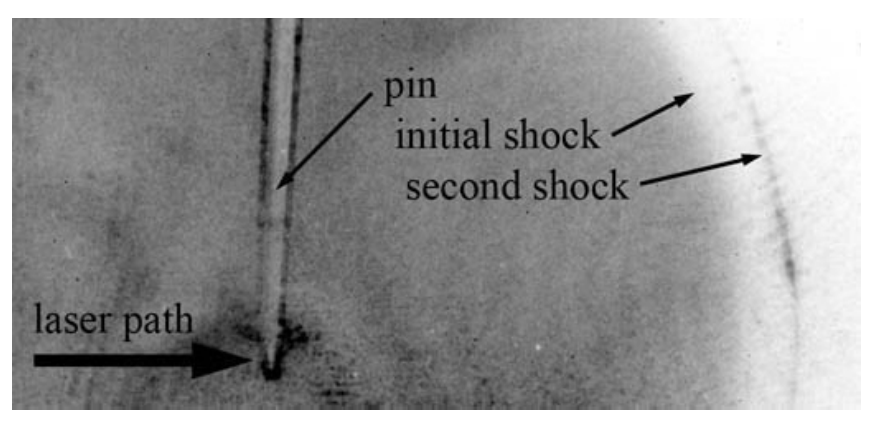

FIG. 2: Experiment set-up: A high-energy infrared laser pulse ablates a small amount of pin material (stainless steel) which expands rapidly, pushing at a surrounding gas (mixture of Xe and $\mathrm{N}_{2}$ ) setting up an initial shock. The initial shock drives a radiative heat wave ahead of it, which in turn creates a second shock. 


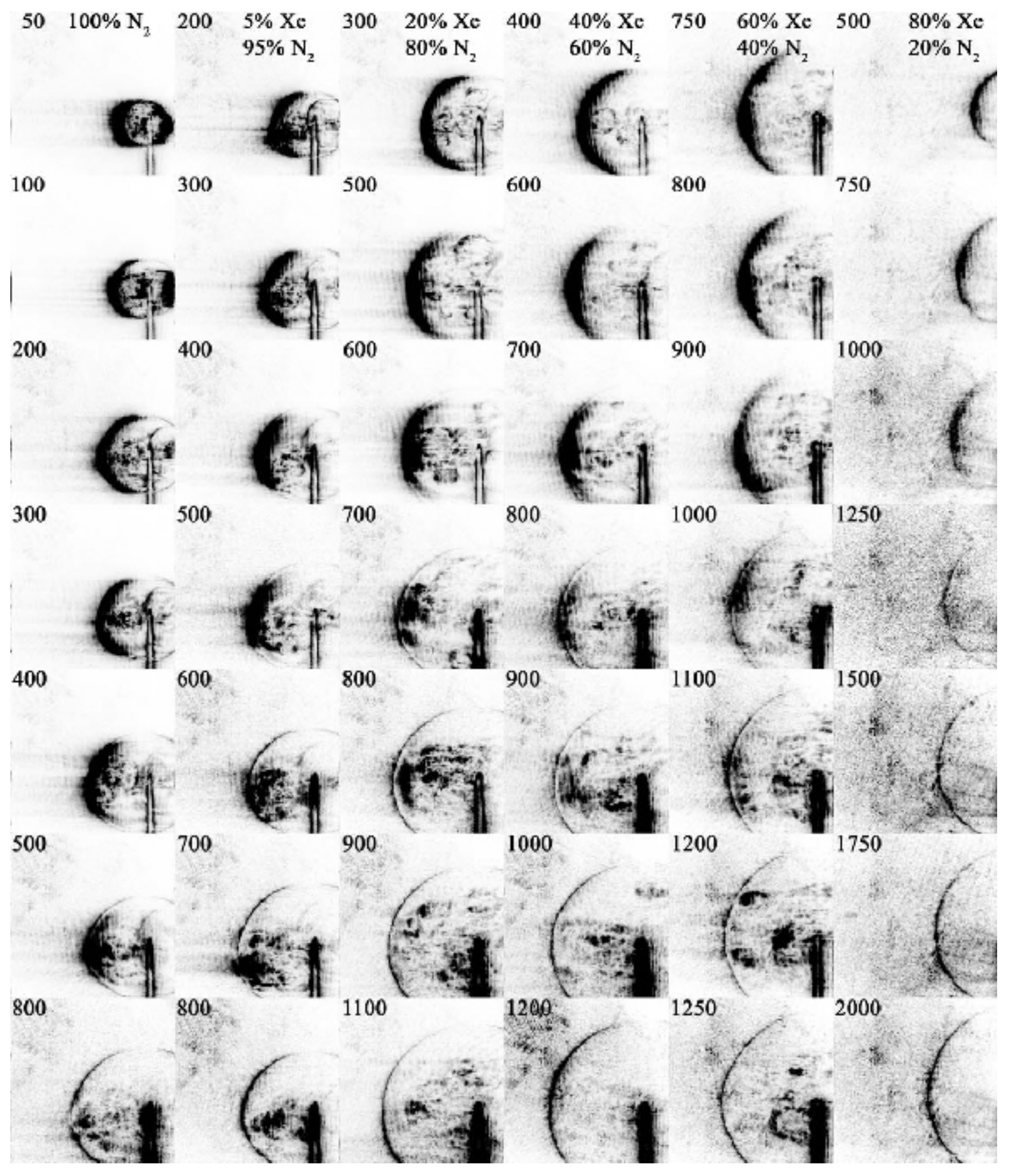

FIG. 3: Images of shocks in Xe/ $\mathrm{N}_{2}$ mixtures with a density $\rho_{0}=3.6 \times 10^{-5} \mathrm{~g} / \mathrm{cm}^{3}$ created by a laser focused on a pin (visible in most images). The mixture is the same within each column of images, and the mass-fraction of each gas is written at the top of the column. The number at the top left of each image is the experimental time in nanoseconds (after the laser pulse). 


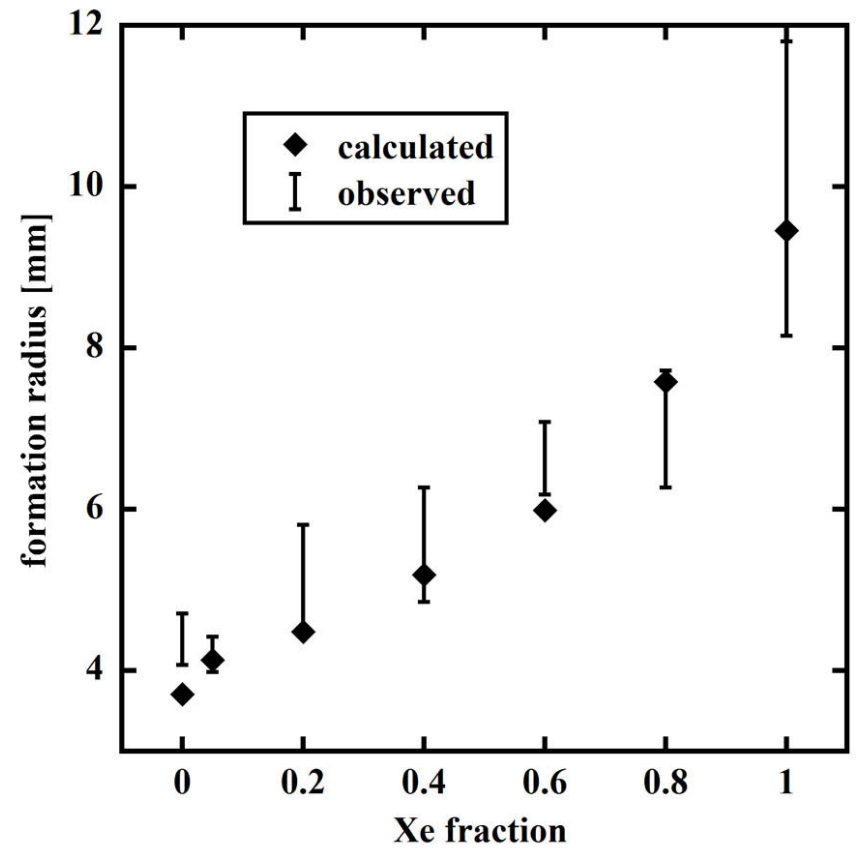

FIG. 4: Calculated and experimentally observed second shock formation radii as a function of Xe mass fraction in Xe/ $\mathrm{N}_{2}$ mixtures. The lower/upper end of each bar represents an image where the second shock cannot/can be seen. 